\title{
EMBEDDINGS BETWEEN WEIGHTED LOCAL MORREY-TYPE SPACES AND WEIGHTED LEBESGUE SPACES
}

\section{R. Ch. Mustafayev and T. ÜnVER}

Abstract. In this paper, the embeddings between weighted local Morrey-type spaces and weighted Lebesgue spaces are investigated.

Mathematics subject classification (2010): 42B35, 47B38.

Keywords and phrases: local Morrey-type spaces, weighted Lebesgue spaces, Hardy-type inequalities.

\section{REFERENCES}

[1] V. I. BuRENKOV, Recent progress in studying the boundedness of classical operators of real analysis in general Morrey-type spaces. I, Eurasian Math. J. 3, 3 (2012), 11-32.

[2] V. I. BuRENKOV, Recent progress in studying the boundedness of classical operators of real analysis in general Morrey-type spaces. II, Eurasian Math. J. 4, 1 (2013), 21-45.

[3] V. I. Burenkov, M. L. Goldman, Necessary and sufficient conditions for boundedness of the maximal operator from Lebesgue spaces to Morrey-type spaces, Math. Inequal. Appl. 17, 2 (2014), $401-418$.

[4] V. I. Burenkov, H. V. Guliyev, Necessary and sufficient conditions for the boundedness of the maximal operator in local spaces of Morrey type, (Russian) Dokl. Akad. Nauk 391, 5 (2003), 591594.

[5] V. I. Burenkov, H. V. Guliyev, Necessary and sufficient conditions for boundedness of the maximal operator in local Morrey-type spaces, Studia Math. 163, 2 (2004), 157-176.

[6] V. I. BurenKov, H. V. GuliYeV, V. S. GuliYeV, Necessary and sufficient conditions for the boundedness of fractional maximal operators in local Morrey-type spaces, J. Comput. Appl. Math. 208, 1 (2007), 280-301.

[7] V. I. Burenkov, H. V. Guliyev, V. S. Guliyev, On boundedness of the fractional maximal operator from complementary Morrey-type spaces to Morrey-type spaces, The interaction of analysis and geometry, Contemp. Math. 424, Amer. Math. Soc., Providence, RI, 2007, 17-32.

[8] V. I. Burenkov, V. S. Guliyev, A. Serbetci, T. V. Tararykova, Necessary and sufficient conditions for the boundedness of genuine singular integral operators in local Morrey-type spaces, Eurasian Math. J. 1, 1 (2010), 32-53.

[9] V. I. Burenkov, A. Gogatishvili, V. S. Guliyev, R. Ch. Mustafayev, Boundedness of the fractional maximal operator in local Morrey-type spaces, Complex Var. Elliptic Equ. 55, 8-10 (2010), 739-758.

[10] V. I. Burenkov, A. Gogatishvili, V. S. Guliyev, R. Ch. Mustafayev, Boundedness of the Riesz potential in local Morrey-type spaces, Potential Anal. 35, 1 (2011), 67-87.

[11] V. I. Burenkov, P. Jain, T. V. TARARYKova, On boundedness of the Hardy operator in Morreytype spaces, Eurasian Math. J. 2, 1 (2011), 52-80.

[12] V. I. Burenkov, E. D. Nursultanov, Description of interpolation spaces for local Morrey-type spaces, (Russian, with Russian summary) Teoriya Funktsii i Differentsialnye Uravneniya, Tr. Mat. Inst. Steklova 269 (2010), 52-62. Translation: Proc. Steklov Inst. Math. 269, 1 (2010), 46-56.

[13] M. Christ, L. Grafakos, Best constants for two nonconvolution inequalities, Proc. Amer. Math. Soc. 123, 6 (1995), 1687-1693.

[14] P. DrÁbeK, H. P. HeInig, A. Kufner, Higher-dimensional Hardy inequality, General inequalities, 7, Oberwolfach 1995, Internat. Ser. Numer. Math. 123, Birkhäuser, Basel, 1997, 3-16. 
[15] A. Gogatishvili, R. Ch. Mustafayev, Dual spaces of local Morrey-type spaces, Czechoslovak Math. J. 61(136), 3 (2011), 609-622.

[16] A. Gogatishvili, R. Ch. Mustafayev, The multidimensional reverse Hardy inequalities, Math. Inequal. Appl. 15, 1 (2012), 1-14.

[17] A. Gogatishvili, R. Ch. Mustafayev, New pre-dual space of Morrey space, J. Math. Anal. Appl. 397, 2 (2013), 678-692.

[18] V. S. GuliYeV, Function spaces, integral operators and two weighted inequalities on homogeneous groups. Some applications, Elm, Baku, 1999 (Russian).

[19] V. S. Guliyev, R. CH. MustafaEv, Integral operators of potential type in spaces of homogeneous type, (Russian) Dokl. Akad. Nauk 354, 6 (1997), 730-732.

[20] V. S. Guliyev, R. CH. Mustafaev, Fractional integrals in spaces of functions defined on spaces of homogeneous type, (Russian, with English and Russian summaries) Anal. Math. 24, 3 (1998), 181200.

[21] A. Kufner, L.-E. Persson, Weighted inequalities of Hardy type, World Scientific Publishing Co. Inc., River Edge, NJ, 2003.

[22] B. Muckenhoupt, Weighted norm inequalities for the Hardy maximal function, Trans. Amer. Math. Soc. 165 (1972), 207-226.

[23] B. Opic, A. Kufner, Hardy-type inequalities, Pitman Research Notes in Mathematics Series, 219, Longman Scientific \& Technical, Harlow, 1990.

[24] W. Rudin, Principles of mathematical analysis, Second edition, McGraw-Hill Book Co., New York, 1964. 